\title{
Aprendizagem organizacional em bibliotecas acadêmicas: uma revisão sistemática
}

Marouva Fallgatter Faqueti

Bibliotecária no Instituto Federal Catarinense, Doutoranda no Programa de Pós-Graduação de Engenharia e Gestão do Conhecimento, UFSC

João Bosco da Mota Alves

Docente, doutor no Programa de Pós-Graduação de Engenharia e Gestão do Conhecimento, UFSC

Andrea Valéria Steil

Docente, doutora no Programa de Pós-Graduação de Engenharia e Gestão do Conhecimento e no Programa de Pós-Graduação em Psicologia, UFSC

http://dx.doi.org/10.1590/1981-5344/2699

O tema Aprendizagem Organizacional (AO) em bibliotecas acadêmicas no Brasil é pouco explorado e as publicações que apresentam resultados de estudos empíricos são incipientes. Considerando a importância de aprofundar os estudos na área, foi realizada uma revisão sistemática na literatura visando identificar e analisar como o tema de $A O$, relacionado ao contexto das bibliotecas acadêmicas, vem sendo abordado em estudos empíricos no período de 2005 a 2015. Para a análise, foram selecionados 16 artigos. As áreas temáticas abordadas nos estudos se concentram em: AO enquanto processo (aprendizagem individual, grupal e organizacional); relacionamentos da AO com a gestão do conhecimento; gestão da informação e avaliação institucional; impactos da cultura organizacional sobre $A O$ e capacidade de aprendizagem organizacional. Quanto aos procedimentos metodológicos utilizados, o uso de abordagens quantitativas prevalece. A síntese dos resultados descritos nos artigos aponta para: a) a relevância da cultura da aprendizagem, da cultura organizacional e do estilo da liderança para a $A O$ e o desempenho das bibliotecas; b) a importância da aprendizagem individual e grupal para se alcançar a $A O$; c) as comunidades de aprendizagem (em redes intra $e$ interdepartamental) e comunidades de prática como significativas para gerar e transferir conhecimentos 
adquiridos; d) baixa AO a partir de processos de avaliação institucional.

Palavras-chave: Aprendizagem organizacional; Biblioteca acadêmica; Biblioteca universitária; Revisão sistemática; Bibliometria.

\section{Organizational Learning in Academic Libraries: A Systematic Review}

The Organizational Learning (OL) theme in academic libraries in Brazil is underexplored and publications presenting results of empirical studies are incipient. Considering the importance of further study in the area, a systematic review was conducted in the literature to identify and analyze how the subject of $O L$, related to the context of academic libraries, has been approached in empirical studies from 2005 to 2015. For analysis, 16 articles were selected. Subject areas addressed in the studies focus on: $O L$ as a process (individual, group and organizational learning); OL relationships with knowledge management; information management and institutional assessment; impact of organizational culture on $O L$ and organizational learning capacity. Related to the methodological approach, prevalent quantitative studies. A summary of the results described in the articles points to: a) the relevance of the learning culture, organizational culture and leadership style to the $O L$ and the performance of libraries; b) the importance of individual and group learning to achieve the $O L ; c)$ the learning communities (intra and interdepartmental networks) and communities of practice as significant to generate and transfer knowledge acquired; d) Low OL from institutional assessment processes.

Keywords: Organizational learning; Academic library; Systematic review; Bibliometrics.

Recebido em 02.02.2016 Aceito em 26.07.2016 


\section{Introdução}

Bibliotecas acadêmicas, também denominadas bibliotecas universitárias, são unidades organizacionais pertencentes a instituições que ofertam cursos de nível superior. Sua estrutura enquanto unidade organizacional dentro de uma instituição (pública ou privada) que atende um público determinado, necessita estar em constante melhoria de seu desempenho e inovando em seus serviços e produtos. Novas tecnologias, mudanças nas expectativas dos usuários/clientes e novos desafios no contexto educativo são alguns dos motivos que promovem a busca contínua por mais conhecimentos.

Em ambientes desta natureza a aprendizagem é um fator estratégico para o desenvolvimento e aperfeiçoamento de competências individuais, grupais e organizacionais a fim de alcançar melhorias no seu desempenho e inovar. Como afirma Lubisco (2014), há uma preocupação por parte das bibliotecas universitárias de estarem na vanguarda técnica e tecnológica na gestão de seus serviços e produtos. Sendo assim, estudos sobre como ocorrem os processos de aprendizagem organizacional em instituições de ensino, e mais especificamente em suas bibliotecas, são necessários para ampliar a compreensão e melhor fundamentar futuras práticas.

Aprendizagem organizacional $(\mathrm{AO})$ é uma área relativamente recente no ambiente acadêmico. As pesquisas iniciais datam da década de 1960. Steil (2002) narra que Cyert e March (1963) foram os primeiros a articularem que as organizações poderiam aprender de maneiras distintas dos indivíduos. Em 1965 surge a primeira publicação com o termo "aprendizagem organizacional" no título, escrita por Cangelosi e Dill. Argyris e Shon, em 1978, sistematizaram os achados da área até então (STEIL, 2002). Outros estudos se seguiram, mas foi na década de 90 que houve a popularização do tema na comunidade científica. Um dos marcos deste período foi a publicação de uma edição especial do periódico Organization Science sobre o tema de AO, contendo artigos dos principais pesquisadores sobre o tema na época.

No contexto de estudos de $\mathrm{AO}$ em Bibliotecas pode-se inferir que a temática se encontra em fase inicial de desenvolvimento. $O$ artigo mais antigo recuperado em buscas nas bases de dados da Web of Science e Scopus, utilizando-se os termos Orgnizational learning e library, data de 1992 (EVALD, 1992), relativo a bibliotecas públicas. No contexto de bibliotecas acadêmicas a primeira publicação recuperada data de 1998 (FOWLER, 1998), sendo que a partir de 2006 percebe-se uma ampliação no número de publicações sobre o tema no nível internacional.

No Brasil, estudos que relacionem $\mathrm{AO}$ em bibliotecas são incipientes. Foi localizado na literatura duas revisões com foco em gestão do conhecimento que apresentam algumas citações referente a $\mathrm{AO}$, como é o caso do estudo de Bem e Coelho (2013). As pesquisadoras identificaram dois artigos abrangendo $\mathrm{AO}$, sendo um direcionado em como as 
organizações podem se transformar em organizações de aprendizagem e outro voltado para inovação e cultura em bibliotecas públicas. A outra revisão encontrada foi realizada por Costa e Castro (2014) apresentando um mapeamento temático sobre gestão do conhecimento (GC) em bibliotecas no Brasil, entre 2000 e 2013. Segundo as autoras o tema AO em bibliotecas ainda é pouco explorado no contexto da GC.

Diante dessa realidade detecta-se a necessidade de se investir em pesquisas na área, porém considera-se relevante conhecer e mapear os estudos empíricos já realizados. Sendo assim, o objetivo deste artigo é analisar como o tema de $A O$, relacionado ao contexto das bibliotecas acadêmicas, vem sendo abordado em estudos empíricos na literatura nacional e internacional.

$\mathrm{Na}$ sequência, o artigo apresenta os fundamentos teóricos relacionados aos temas abordados, descreve os procedimentos metodológicos e por fim discorre sobre os resultados alcançados e conclusões obtidas.

\section{Aprendizagem organizacional}

Estudos na área de Aprendizagem Organizacional (AO) emergiram na década de 60 e se multiplicaram na década de 90 (ANTONELLO, GODOY, 2011). O crescimento segue multidisciplinarmente abrangendo áreas do conhecimento como: psicologia e desenvolvimento organizacional; ciência administrativa; sociologia e teoria organizacional; estratégia; gerenciamento da produção; antropologia cultural, dentre outras (ANTAL et al., 2001; EASTERBY-SMITH, 1997; BONTIS; CROSSAN; HULLAND, 2002).

Conceitualmente $\mathrm{AO}$ permanece em continua reconstrução. Para efeito deste trabalho entende-se a $\mathrm{AO}$ como "[...] um processo, uma construção social que transforma o conhecimento criado pelo indivíduo em ações institucionalizadas em direção aos objetivos organizacionais" (ANGELONI; STEIL, 2011, p. 121).

Destaca-se, que pesquisas sobre aprendizagem em organizações revelam o uso de dois termos: "aprendizagem organizacional" (AO) e "organizações de aprendizagem" (OA), utilizados por alguns autores como sinônimos. Entretanto existe uma clara distinção entre eles (TSANG, 1997). Estudos em AO buscam descrever e compreender processos e comportamentos, pautados no rigor científico (visão descritiva). Escritos de OA, no entanto se propõem a apresentar um estado ideal, recomendações, prescrições, muitas vezes com base em experiências de consultoria e melhores práticas (visão prescritiva). Para clarear essa distinção resgata-se a definição de OA de Garvin (1993), como aquelas organizações que possuem a capacidade de adquirir, criar, produzir novos insights, transferir conhecimentos e modificar o comportamento de seus membros.

Diferentes abordagens sobre aprendizagem nas organizações são encontradas na literatura, dentre as quais destacam-se: 
a) aquelas que focam na aprendizagem como um processo, ou na aprendizagem como um resultado/produto (conhecimento) (EASTERBY-SMITH; LYLES, 2005);

b) abordagens com uma ênfase maior em um nível de análise ou em vários níveis de aprendizagem ao mesmo tempo, como o indivíduo, o grupo, a organização, as relações entre a organização com outras organizações e as redes organizacionais (SNYDER, CUMMINGS, 1998; LEVINSTON. ASAHI, 1995; KNIGHT, 2002; CROSSAN et al., 1995);

c) abordagens descritivas, como a aprendizagem organizacional, e abordagens prescritivas, como foco no desenvolvimento de um tipo ideal de organização (organização de aprendizagem) (TSANG, 1997; EASTERBY-SMITH; LYLES 2005);

d) por fim, abordagens que focam os processos de aprendizagem como uma mudança cognitiva, uma mudança comportamental, ou um processo que envolve tantas mudanças cognitivas, quanto comportamentais (INKPEN; CROSSAN, 1995).

Para além das abordagens descritas, Antonello (2005) sugere a existência de seis focos mais abordados na literatura de $A O$ : foco na mudança; foco na socialização da aprendizagem individual; foco em processo-sistema; foco na cultura; foco no gerenciamento do conhecimento; foco em melhoria continua e foco em inovação.

Pode-se ainda identificar que a $A O$ tem sido relacionada a construtos que também focam na criação, disseminação, absorção e institucionalização de conhecimentos, como a gestão do conhecimento (POLANYI, 1966), o conhecimento organizacional (SANTOS; STEIL, 2015, LAVE; WENGER, 1991), a memória organizacional (CASEY; OLIVERA, 2011; STEIL; SANTOS, 2012), a desaprendizagem organizacional (TSANG, ZAHRA, 2008), a capacidade absortiva (ZAHRA; GEORGE, 2002) e a capacidade de aprendizagem (GOH; RICHARDS, 1997).

\section{Bibliotecas acadêmicas}

Bibliotecas são criadas com a missão de atender as necessidades informacionais e educacionais de um determinado público. Com base nesse público desenvolvem-se os serviços que visam atender as necessidades da demanda. As bibliotecas acadêmicas, podem ser entendidas como unidades organizacionais pertencentes a uma instituição de ensino superior (universidade, faculdade, instituto tecnológico, dentre outros), que prestam serviços à comunidade interna composta por discentes, docentes, servidores técnicos administrativos e, por extensão, à comunidade local. Segundo Lubisco (2014), sua função é respaldar o 
desenvolvimento institucional relacionado aos programas de ensino, pesquisa, extensão e inovação. Suas atividades se desenvolvem tradicionalmente sob três focos: serviços aos usuários, serviços administrativos e serviços técnicos. No entanto, atualmente suas tarefas têm sido desafiadas por mudanças cada vez mais complexas e constantes nos ambientes organizacional, tecnológico e informacional (KASSIM; SHOID, 2013).

Pertencentes a instituições de ensino superior, reconhecidas como organizações intensivas de conhecimento (SOUZA, 2009; TAKARASHI, 2007), as bibliotecas igualmente podem ser percebidas como unidades organizacionais que possuem atividades intensivas em conhecimento (RIBEIRO JR; COSTA, 2013). Bibliotecários repensam seus processos de gestão tradicional de bibliotecas abrindo-se em busca de novos caminhos. Estudos na área de gestão do conhecimento aplicada a bibliotecas têm se intensificado. Estes estudos têm focado nos ativos intagíveis que facilitam o desempenho mais eficiente e eficaz dos bibliotecários em suas funções (JANTZ, 2001) e para promover a inovação (SHANHONG, 2000) e melhoria no desempenho organizacional.

Intensificar pesquisas no sentido de melhor compreender os processos de aprendizagem e de conhecimento no campo das bibliotecas é um caminho promissor, posto que, segundo Fowley (1998) este são diferenciais para a sua sobrevivência acompanhando as mudanças externas, inovando e cumprindo assim o seu papel na instituição.

\section{Procedimentos metodológicos}

Entende-se que Revisão Sistemática implica na "[...] aplicação de estratégias científicas que limitem o viés de seleção de artigos, avaliem com espírito crítico os artigos e sintetizem todos os estudos relevantes em um tópico específico" (PERISSÉ; GOMES; NOGUEIRA, 2001). Esse tipo de estudo se propõe a sintetizar e analisar o conhecimento cientifico já publicado utilizando uma metodologia passível de ser avaliada e replicada. (BOTELHO; CUNHA; MACEDO, 2011).

Dentro desta perspectiva, a revisão sistemática realizada teve como objetivo geral: analisar como o tema de $\mathrm{AO}$, relacionado ao contexto das bibliotecas de instituições educacionais de ensino superior vem sendo abordado em estudos empíricos, na literatura nacional e internacional. E como os objetivos específicos: a) caracterizar os estudos por meio de análises bibliométricas; b) identificar as principais áreas temáticas; c) identificar fontes de informação mais utilizadas d) descrever os métodos de pesquisa utilizados; e) analisar os objetivos e os principais resultados obtidos.

A busca sistemática na literatura, deu-se no dia 10 de agosto de 2015 e os passos efetuados seguem descritos no Quadro 1: 
Quadro 1 - Descrição dos passos realizados na busca sistemática

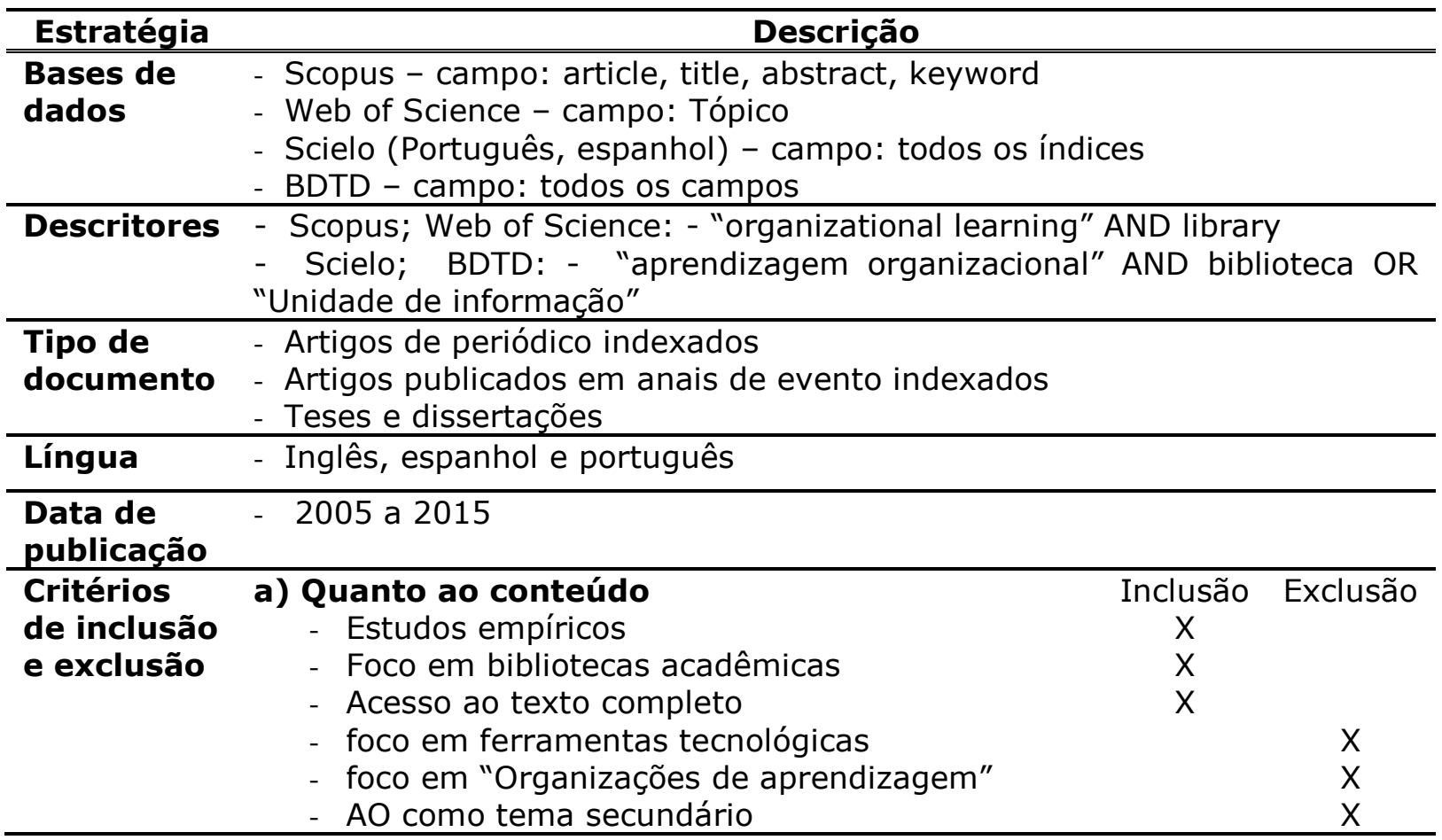

Fonte: Dados da pesquisa. etapas:

O processo de seleção dos trabalhos foi desenvolvido em duas

a) leitura prévia dos títulos e resumos, aplicando-se os filtros de inclusão. Essa etapa gerou o grupo de trabalhos préselecionados;

b) leitura completa das produções pré-selecionadas, aplicando-se os filtros de exclusão.

A síntese dos resultados da pesquisa realizada para seleção dos artigos é apresentada no Tabela 1 a seguir:

Tabela 1 - Síntese dos resultados da pesquisa para seleção dos artigos

\begin{tabular}{lccc}
\hline BASE & RECUPERADOS & PRÉ-SELECIONADOS & SELECIONADOS \\
\hline \hline Web of Science & 25 & 16 & 6 \\
\hline Scopus & 39 & 23 & 9 \\
\hline Scielo & 2 & 1 & 1 \\
\hline BDTD & 2 & 0 & 0 \\
\hline TOTAL & 68 & 30 & 16 \\
\hline
\end{tabular}

Fonte: Dados da pesquisa. 
No Quadro 2 a seguir apresenta-se a descrição de autoria, título e ano das publicações dos 16 artigos selecionados:

Quadro 2 - Publicações selecionadas para análise

\begin{tabular}{|c|c|c|c|}
\hline Id & Ano & Autoria & Título \\
\hline 1 & 2015 & $\begin{array}{l}\text { Yu, T.; Chen, } \\
\text { C. C }\end{array}$ & $\begin{array}{l}\text { The relationship of learning culture, learning method, and } \\
\text { organizational performance in the university and college libraries in } \\
\text { Taiwan }\end{array}$ \\
\hline 2 & 2014 & $\begin{array}{l}\text { Shoid, M. S. } \\
\text { M.; Kassim, } \\
\text { N.A. }\end{array}$ & $\begin{array}{l}\text { Using regression analysis on measuring organizational learning } \\
\text { capabilities (OLC) dimensions" }\end{array}$ \\
\hline 3 & 2014 & $\begin{array}{l}\text { Shoid, M. S. } \\
\text { M.; Kassim, N. } \\
\text { A. }\end{array}$ & $\begin{array}{l}\text { Exploring the effect of organizational learning capabilities (OLC) on } \\
\text { knowledge performance }\end{array}$ \\
\hline 4 & 2013 & $\begin{array}{l}\text { Kassim, N. A.; } \\
\text { Shoid, M.S.M }\end{array}$ & $\begin{array}{l}\text { Organizational learning capabilities and knowledge performance in } \\
\text { university technology Mara (UTM) Library, Malaysia }\end{array}$ \\
\hline 5 & 2013 & $\begin{array}{l}\text { Shoid, M. S. } \\
\text { M.; Kassim, } \\
\text { N. A. }\end{array}$ & $\begin{array}{l}\text { Ascertaining dimensions of organizational learning capabilities } \\
\text { (OLC) in academic library }\end{array}$ \\
\hline 6 & 2013 & $\mathrm{Yu}, \mathrm{T}$ & $\begin{array}{l}\text { The relationships among individual, team and organizational } \\
\text { learning in Taiwan's university and college libraries }\end{array}$ \\
\hline 7 & 2012 & $\begin{array}{l}\text { Shoid, M. S. } \\
\text { M.; Kassim, N. } \\
\text { A. }\end{array}$ & $\begin{array}{l}\text { Organizational culture and teamwork cooperation as determinants } \\
\text { of organizational learning capabilities (OLC) in academic library }\end{array}$ \\
\hline 8 & 2012 & $\begin{array}{l}\text { Yu, T.; Chen, } \\
\text { C. C; }\end{array}$ & $\begin{array}{l}\text { A Study on the relationship between organizational learning culture } \\
\text { and organizational performance in Taiwan's university and college } \\
\text { libraries }\end{array}$ \\
\hline 9 & 2011 & Dewi, I. C. & $\begin{array}{l}\text { Organizational learning and transformational leadership in higher } \\
\text { education }\end{array}$ \\
\hline 10 & 2008 & $\begin{array}{l}\text { Chen, } \mathrm{K} . ; \text { Lin, } \\
\text { P. C. }\end{array}$ & $\begin{array}{l}\text { Organizational learning in the evaluation procedures: a qualitative } \\
\text { study }\end{array}$ \\
\hline 11 & 2008 & Duarte, et al. & $\begin{array}{l}\text { Aprendizagem organizacional em unidades de informação: do grupo } \\
\text { focal à comunidade de prática. }\end{array}$ \\
\hline 12 & 2008 & $\begin{array}{l}\text { Abdullah, K. A. } \\
\text { S.; Kassim, N. } \\
\text { A. }\end{array}$ & $\begin{array}{l}\text { Perceptions of organizational learning practices among yemeni } \\
\text { university librarians }\end{array}$ \\
\hline 13 & 2007 & Chen, $\mathrm{K}$ & Institutional evaluation and its influence on organizational learning \\
\hline 14 & 2007 & $\begin{array}{l}\text { Kassim, N. A.; } \\
\text { Nor, A. M. }\end{array}$ & $\begin{array}{l}\text { Team learning in a learning organization: the practices of team } \\
\text { learning among university librarians in Malaysia }\end{array}$ \\
\hline 15 & 2006 & Chen, $\mathrm{K}$. & $\begin{array}{l}\text { Library evaluation and organizational learning: a questionnaire } \\
\text { study }\end{array}$ \\
\hline 16 & 2006 & $\begin{array}{l}\text { Figueroa, L. } \\
\text { A .; Gonzalez, } \\
\text { A. B. }\end{array}$ & $\begin{array}{l}\text { Management of knowledge, information and organizational learning } \\
\text { in university libraries }\end{array}$ \\
\hline
\end{tabular}

Fonte: Dados da pesquisa.

A terceira etapa contemplou o processo de análise aprofundada dos trabalhos selecionados. Para tanto, organizou-se uma matriz síntese com os conteúdos a serem analisados conforme os objetivos propostos. Os 
resultados alcançados seguem descritos no próximo capítulo. Apresentamse sequencialmente análises contextuais bibliométricas dos artigos, seguido de análises qualitativas e considerações finais.

\section{Indicadores bibliométricos}

Visando facilitar a contextualização em relação aos estudos selecionados optou-se por retratar alguns dados bibliométricos. Para iniciar apresentam-se os dados referentes à autoria, periódicos e datas de publicação dos artigos. Em seguida mostram-se um quadro referente à localização geográfica das produções e um mapeamento dos principais temas abordados nos trabalhos.

\subsection{Quanto a autoria, periódicos e datas de publicação}

No que se refere à autoria dos artigos, a maioria dos estudos (12 artigos) possui autoria múltipla, entre 2 a 6 autores. Pesquisadores de origem asiática se destacam pelo número de publicações como autores ou co-autores: Norliya Ahmad Kassim - 7 artigos; Mohd Shamsul Mohd Shoid - 5 artigos; Kuan-Nien Chen - 3 artigos; Ti Yu - 3 artigos; Chen ChaoChen - 2 artigos. Dentre os 20 autores, 11 publicaram apenas uma vez sobre o tema.

Em relação aos periódicos observou-se que os artigos foram publicados em revistas das áreas de ciências Sociais e interdisciplinares: Aslib Proceedings New Information Perspectives; College \& Research Libraries; International Journal of Academic Research In Business and Social Sciences; Journal of Educational Media and Library Science; Journal of Librarianship and Information Science; Libri; Malaysian Journal of Library and Information Science; Perspectivas em Ciência da Informação; Middle - East Journal of Scientific Research ; World Applied Sciences Journal. Alguns artigos foram publicados em eventos internacionais, como: IFLA Wlic 2013; Symposium on Business; Engineering and Industrial Applications $e$, Proceedings of the 8th International Conference on Intellectual Capital, Knowledge Management And Organisational Learning.

Quanto aos anos de publicação enquadrados nos limites desta pesquisa (2005 a 2015), percebe-se que até 2010 houve 7 publicações e entre 2010 a 2015 foram publicados 9 artigos. Observa-se assim, que existe uma tendência crescente de publicações na área nos últimos anos.

\subsection{Quanto a localização geográfica}

Quanto à localização geográfica dos estudos, a maioria foi desenvolvida no continente asiático (Figura 1). As pesquisas mais recentes foram realizadas em Taiwan e Malásia.

a) Yemen, Ásia ocidental - 1 estudo de (ABDULLAH; KASSIM, 2008); 
b) Malásia, Ásia oriental - 2 estudos, sendo que os resultados foram apresentados em 5 artigos (SHOID; KASSIM, 2012); (KASSIM; SHOID, 2013); (SHOID; KASSIM, 2013); (SHOID; KASSIM, 2014a); (SHOID; KASSIM, 2014b)

c) Indonésia, Ásia oriental - 1 estudo de Dewi (2011)

d) Taiwan (Republica da China) Ásia oriental - 3 estudos com resultados apresentados em 6 artigos (CHEN, 2006; 2007); (CHEN; LIN, 2008); (YU; CHEN, 2012) (YU, 2013) (YU; CHEN, 2015);

e) Brasil, América do Sul - 1 estudo realizado por Duarte et al. (2008);

f) Chile, América do Sul - 1 estudo realizado por Figueroa e Gonzalez (2006).

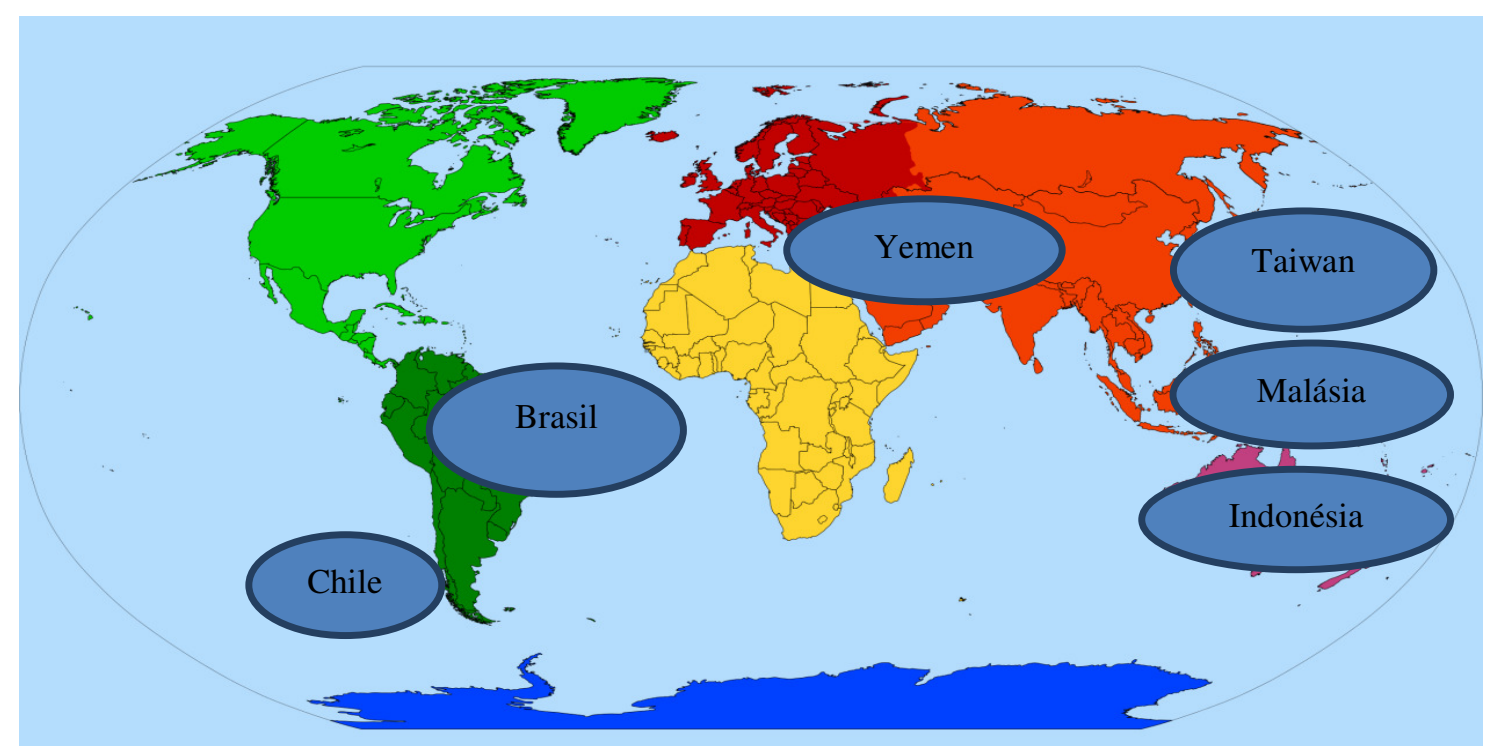

Figura 1 - Localização geográfica das publicações

Fonte: Elaborado pelos autores.

\subsection{Quanto as fontes de informação referenciadas}

A análise das fontes de informação referenciadas nos artigos permite observar quais autores compõem o corpo teórico basilar que fundamenta as pesquisas realizadas. A tabela 2 apresenta as obras citadas no mínimo 4 vezes (presença em $25 \%$ dos artigos) dentro do universo das 405 referências descritas nos 16 artigos (média de 25,12 obras por artigo). 
Tabela 2 - Obras mais referenciadas e seus temas correspondentes

\begin{tabular}{|c|c|c|c|}
\hline Autor / título & Temas & Citações & Tipo \\
\hline $\begin{array}{l}\text { SENGE, P. The Fifth Discipline: the art and practice of the } \\
\text { learning organisation. New York: Doubleday, } 1990 .\end{array}$ & $\mathrm{OA}$ & 11 & Livro \\
\hline $\begin{array}{l}\text { IMRAN, M.; RIZVI, S. H. M.; ALI, B. Impact of organizational } \\
\text { learning on organizational performance. International Journal of } \\
\text { Academic Research, v. } 3, \text { n. 4, p. 424-427, } 2011 .\end{array}$ & $\begin{array}{c}\mathrm{AO} \text { e } \\
\text { desempenho }\end{array}$ & 6 & Artigo \\
\hline $\begin{array}{l}\text { CHEN, K. N. Library evaluation and organizational learning: a } \\
\text { questionnaire study. Journal of Librarianship and Information } \\
\text { Science, v. } 38, \text { n. 2, p. } 93-104,2006 \text {. }\end{array}$ & $\begin{array}{l}\text { AO em } \\
\text { avaliação de } \\
\text { bibliotecas }\end{array}$ & 5 & Artigo \\
\hline $\begin{array}{l}\text { WATKINS, K. E.; MARSICK, V. J. Sculpting the learning } \\
\text { organization: lessons in the art and science of systemic change. } \\
\text { San Francisco: Jossey-Bass Inc, } 1993 .\end{array}$ & $\mathrm{OA}$ & 5 & Livro \\
\hline $\begin{array}{l}\text { ARGYRIS, C.; SCHON, D. A. Organizational Learning: a Theory of } \\
\text { Action Perspective. Reading, MA: Addison-Wesley, } 1978 .\end{array}$ & $\mathrm{AO}$ & 4 & Livro \\
\hline $\begin{array}{l}\text { DODGSON, M. Organizational learning: a review of some } \\
\text { literatures. Organization Studies, v. 14, n. 3, p. 375-394, } 1993 .\end{array}$ & $\mathrm{AO}$ & 4 & Artigo \\
\hline $\begin{array}{l}\text { PRESKILL, H. Evaluation's role in enhancing organizational } \\
\text { learning. Evaluation and Program Planning, v. 17, n. 3, p. 291- } \\
\text { 297, } 1994 \text {. }\end{array}$ & $\begin{array}{c}\text { AO e } \\
\text { avaliação }\end{array}$ & 4 & Artigo \\
\hline $\begin{array}{l}\text { ROWLEY, J. The library as a learning organization. Library } \\
\text { Management, } v .18, \text { n. } 2, \text { p. } 88-91,1997 .\end{array}$ & $\begin{array}{c}\text { OA em } \\
\text { bibliotecas }\end{array}$ & 4 & Artigo \\
\hline $\begin{array}{l}\text { GOH, S. C.; RICHARDS, G. (1997). Benchmarking the learning } \\
\text { capability of organisations. European Management Journal, v. } \\
15, \text { n. 5, p. } 575-583,1997 \text {. }\end{array}$ & Cap. de AO & 4 & Artigo \\
\hline $\begin{array}{l}\text { MARSICK, V. J.; WATKINS, K. E. 2003. Demonstrating the Value } \\
\text { of an Organization's Learning Culture. Advances in Developing } \\
\text { Human Resources, v. 5, n. 2, p. 132-51, } 2003 .\end{array}$ & AO e cultura & 4 & Artigo \\
\hline
\end{tabular}

Fonte: Dados da pesquisa.

Constata-se que a maioria dos artigos $(11=68,7 \%)$ faz referência a obra Peter Senge, demonstrando uma tendência de estudos sobre bibliotecas como organizações de aprendizagem. Tal foco é reforçado por meio de referências a Watkins, e Marsick (5 citações) e Rowley (4 citações). Em contrapartida, constata-se apenas uma referência (4 citações) para um dos marcos teóricos referente ao contexto específico de AO (Argirys e Shon). Quatro artigos optaram por não citar diretamente as fontes originais sobre $A O$ e sim referenciar um artigo de revisão de Dogson. Outro ponto observado foi o baixo índice de referências relacionadas ao campo metodológico, e a alta diversidade entre as citadas, não havendo nenhuma fonte incluída entre as mais citadas.

Quanto as relações de $A O$ e outros constructos temáticos percebe-se que alguns temas relacionados à desempenho (6 citações), AO em bibliotecas ( 5 citações) se destacam, seguidos dos temas de avaliação, capacidade de aprendizagem organizacional e cultura, cada um com quatro citações. Essa diversidade de constructos pode ser melhor entendida na seção seguinte, que aborda as áreas temáticas abordadas em cada artigo. Denota-se aqui que existe uma tendência de estudos voltados para o desenvolvimento de pesquisas de AO cujos resultados 
possam se integrar a perspectivas de promoverem as bibliotecas acadêmicas como OA.

\subsection{Quanto às áreas temáticas}

As pesquisas realizadas na área de aprendizagem em bibliotecas abrangem diferentes contextos, conforme demonstra a Figura 02, podendo ser agrupados da seguinte forma:

a) AO enquanto processo multinível - individual, grupal e organizacional, (YU, 2013; ABDULLAH; KASSIM, 2008; DUARTE et al., 2008; KASSIM; NOR, 2007);

b) interações da $A O$ com a Gestão do Conhecimento (GC), e Gestão da Informação (GI) (FIGUEROA; GONZALEZ, 2006) e com a Avaliação Institucional (AI) (CHEN, 2006; 2007) (CHEN; LIN, 2008);

c) influências da cultura organizacional (YU; CHEN, 2015; 2012) e do estilo de liderança (DEWI, 2011);

d) capacidade de aprendizagem organizacional (SHOID; KASSIM, 2014a; 2014b; 2013; 2012); (KASSIM; SHOID, 2013)

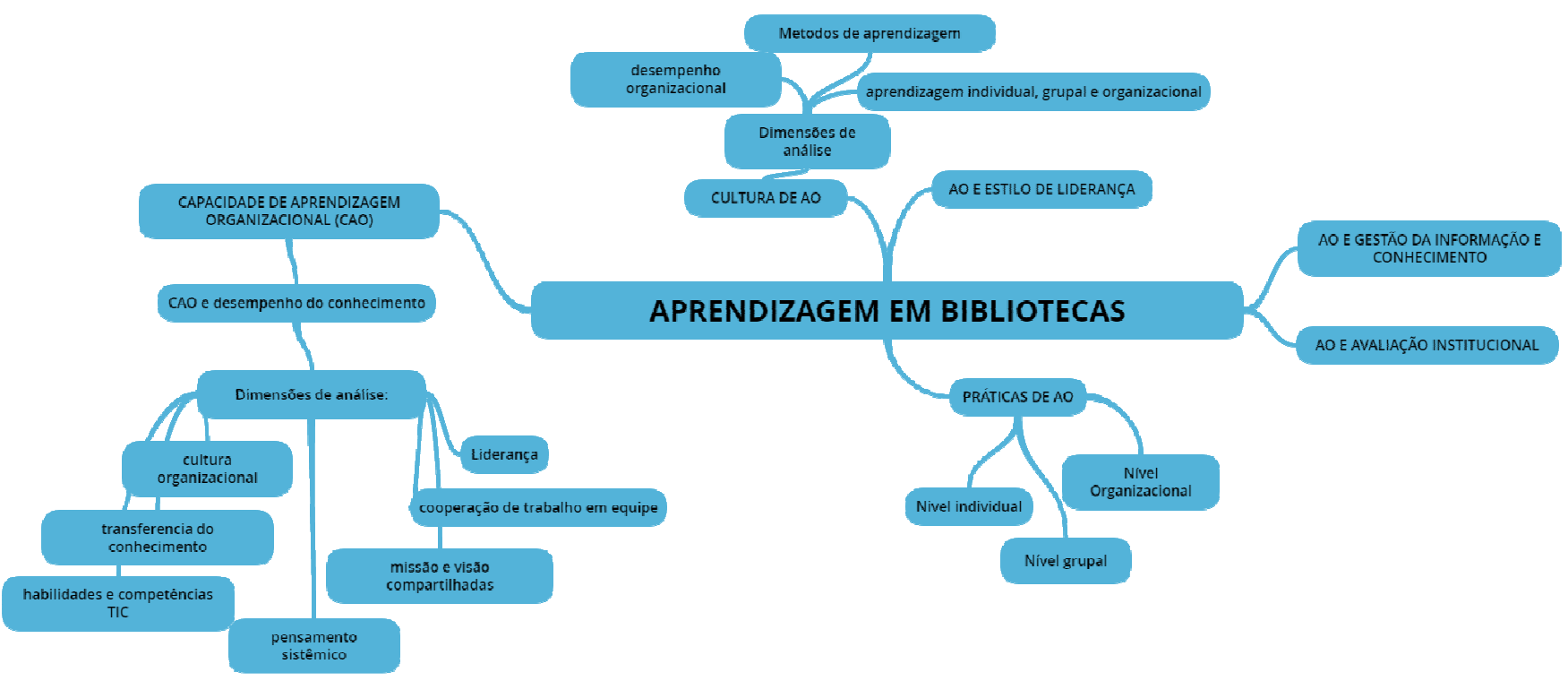

Figura 2 - Mapa temático das pesquisas de AO em bibliotecas

Fonte: Dados da pesquisa. Ferramenta web utilizada: GoConqr. Disponível em: <https://www.goconqr.com>. Acesso em: 10 out. 2015.

A Figura 3 mostra que alguns dos temas estudados correspondem as áreas apontadas por Antonello (2005), quais sejam: aprendizagem 
individual e coletiva, GC, cultura, como referentes a discussões diretas sobre AO.

Foram também encontrados estudos sobre "Capacidade de Aprendizagem Organizacional". Pesquisas nesta área visam identificar as características organizacionais e gerenciais que contribuem para facilitar 0 processo de aprendizagem organizacional (CHIVA; ALEGRE; LAPIEDRA 2007).

Outra temática que se distinguiu das demais é o estudo de AO relacionado a Avaliação Institucional (AI), que visa responder se ocorre AO a partir do processo de AI. Apesar de não discutido desta forma pelos autores, pode-se inferir relação com estudos sobre 'capacidade de absorção de conhecimentos' definido como um conjunto de rotinas e processos organizacionais estratégicos com os quais a empresa adquire, assimila, transforma e explota conhecimento para produzir uma capacidade organizacional dinâmica (ZAHRA; GEORGE, 2002, p. 198, tradução nossa).

\subsection{Quanto aos métodos de pesquisa}

Os 16 artigos selecionados que compõem o universo desta análise de estudos empíricos demonstram uma preferência para utilização da abordagem quantitativa como pode ser identificado no Tabela 3:

Tabela 3 - Métodos de pesquisa utilizados

\begin{tabular}{|c|c|c|c|}
\hline Autoria & Abordagem & Técnica & Público e amostra \\
\hline $\begin{array}{l}\text { Yu, T.; Chen, C. } \\
\text { C (2015) }\end{array}$ & Quantitativa & Questionário & $\begin{array}{l}478 \text { respondentes, representantes de } 162 \\
\text { bibliotecas Universitárias de Taiwan }\end{array}$ \\
\hline $\begin{array}{l}\text { Shoid, M. S. M.; } \\
\text { Kassim, N.A. } \\
(2014 a)\end{array}$ & \multirow[t]{2}{*}{ Quantitativa } & \multirow[t]{2}{*}{ Questionário } & \multirow[t]{2}{*}{$\begin{array}{l}186 \text { bibliotecários de } 8 \text { Universidades da } \\
\text { Malásia }\end{array}$} \\
\hline $\begin{array}{l}\text { Shoid, M. S. M.; } \\
\text { Kassim, N. A. } \\
\text { (2014b) }\end{array}$ & & & \\
\hline $\begin{array}{l}\text { Kassim, N. A.; } \\
\text { Shoid, M.S.M } \\
\text { (2013) }\end{array}$ & \multirow{3}{*}{ Quantitativa } & \multirow{3}{*}{ Questionário } & \multirow{3}{*}{$\begin{array}{l}36 \text { bibliotecários ocupantes de cargos de } \\
\text { nível intermediário e superior e uma } \\
\text { universidade da Malásia (esta descrição } \\
\text { não estava presente nos três artigos, } \\
\text { porém, por meio da análise dos dados dos } \\
\text { respondentes constata-se que os } \\
\text { resultados se referem a uma mesma } \\
\text { pesquisa) }\end{array}$} \\
\hline $\begin{array}{l}\text { Shoid, M. S. M.; } \\
\text { Kassim, N. A. } \\
(2013)\end{array}$ & & & \\
\hline $\begin{array}{l}\text { Shoid, M. S. M.; } \\
\text { Kassim, N. A. } \\
(2012)\end{array}$ & & & \\
\hline $\mathrm{Yu}, \mathrm{T} .(2013)$ & \multirow[b]{2}{*}{ Quantitativa } & \multirow[b]{2}{*}{ Questionário } & \multirow{2}{*}{$\begin{array}{l}478 \text { respondentes (funcionários de } 162 \\
\text { bibliotecas de universidades de Taiwan. }\end{array}$} \\
\hline $\begin{array}{l}\text { Yu, T.; Chen, C. } \\
\text { C; }(2012)\end{array}$ & & & \\
\hline $\begin{array}{l}\text { Dewi, I. C. } \\
\text { (2011) }\end{array}$ & Quantitativa & Questionário & $\begin{array}{l}\text { Bibliotecários de Universidades de } \\
\text { Surabaia, Indonésia. Não informa o } \\
\text { número de respondentes. }\end{array}$ \\
\hline
\end{tabular}




\begin{tabular}{|c|c|c|c|}
\hline P. C. (2008) & \multirow[t]{2}{*}{ Qualitativa } & \multirow{2}{*}{$\begin{array}{l}\text { Entrevista } \\
\text { semiestruturada }\end{array}$} & \multirow{2}{*}{$\begin{array}{l}24 \text { bibliotecários respondentes que atuam } \\
\text { em bibliotecas de Taiwan. }\end{array}$} \\
\hline $\begin{array}{l}\text { Chen, K. N. } \\
(2007)\end{array}$ & & & \\
\hline $\begin{array}{l}\text { Duarte, et al. } \\
(2008)\end{array}$ & Qualitativa & Grupo Focal & $\begin{array}{l}8 \text { Bibliotecários que atuam em Bibliotecas } \\
\text { universitárias. }\end{array}$ \\
\hline $\begin{array}{l}\text { Abdullah, K. A. } \\
\text { S.; Kassin, N. A. } \\
\text { (2008) }\end{array}$ & Quantitativa & Questionário & $\begin{array}{l}198 \text { bibliotecários respondentes de } 18 \\
\text { universidades do Yemen. }\end{array}$ \\
\hline $\begin{array}{l}\text { Kassim, N. A.; } \\
\text { Nor, A. M. } \\
\text { (2007) }\end{array}$ & Quantitativa & Questionário & $\begin{array}{l}191 \text { bibliotecários respondentes de } \\
\text { universidades da Malásia. }\end{array}$ \\
\hline $\begin{array}{l}\text { Chen, K. N. } \\
(2006)\end{array}$ & Quantitativa & Questionário & $\begin{array}{l}\text { Bibliotecários que atuam em } 67 \\
\text { bibliotecas acadêmicas de Taiwan. Não } \\
\text { informa o número de respondentes. }\end{array}$ \\
\hline $\begin{array}{l}\text { Figueroa, L. A .; } \\
\text { Gonzalez, A. } \\
\text { B. (2006) }\end{array}$ & Qualitativa & $\begin{array}{l}\text { Documental e } \\
\text { entrevista em } \\
\text { profundidade }\end{array}$ & $\begin{array}{l}\text { Bibliotecários pertencentes a } 4 \text { bibliotecas } \\
\text { de uma universidade. Não informa o } \\
\text { número de respondentes. }\end{array}$ \\
\hline
\end{tabular}

Fonte: Dados da pesquisa.

\section{Análise qualitativa}

No processo de análise qualitativa primeiramente realizou-se um mapeamento dos objetivos e principais resultados alcançados em cada estudo. Posteriormente os resultados foram reorganizados sinteticamente a partir de categorias que emergiram do processo de análise, permitindo, assim, uma melhor compreensão dos mesmos.

\subsection{Quanto aos objetivos e resultados alcançados}

A proposta de Figueroa e Gonzalez (2006) objetivou, a partir de um estudo qualitativo e documental, caracterizar a gestão da informação, do conhecimento e da aprendizagem organizacional num sistema de bibliotecas universitárias e analisar a relação entre eles. As principais conclusões apontam que os processos dos três elementos estão intimamente ligados e atuam de forma sinergética. Destaca que comunidade de aprendizagem (em redes intra e interdepartamentais) são fundamentais para gerar e transferir conhecimentos adquiridos.

Os estudos sobre Avaliação institucional (AI) e AO apresentados por Chen $(2006$; 2007) e Chen; Lin (2008) objetivaram investigar e descrever a relação entre AI e a resposta organizacional das bibliotecas. No primeiro artigo Chen (2006) propõe um modelo com as possíveis relações entre AO e AI. O modelo gerado derivou de estudos teóricos com embasamento em Argyris e Schon (1978) e Crossan, Lane e White (1999). O Segundo e terceiro artigos (CHEN, 2007; CHEN; LIN, 2008) discorrem sobre os resultados obtidos de uma pesquisa qualitativa que objetivou descrever qualitativamente a natureza da $\mathrm{AO}$ em bibliotecas acadêmicas e, particularmente, explorar o papel da avaliação no processo. Os resultados deste estudo mostram que poucas bibliotecas conseguem alcançar $\mathrm{AO}$ a 
partir da AI. Barreiras internas e problemas estruturais de como a AI é implementada. Parece difícil para algumas bibliotecas usarem a avaliação como uma ferramenta para ajudar na aprendizagem organizacional. Existem dois grandes obstáculos: a) comunicação tradicional top-down e ausência de canais de comunicação mais abertos que facilitem a aprendizagem; b) a natureza ineficaz do AI por sua preocupação com benchmarks de desempenho de curto prazo, e sua exclusão de uma verdadeira parceria organizacional com bibliotecas.

Kassim e Shoid desenvolveram dois estudos sobre a Capacidade de Aprendizagem Organizacional (CAO) e desempenho do conhecimento. Segundo entendimento dos autores CAO é definida como a organização e gestão de características e fatores que incentivam o processo de aprendizagem ou que permitem que uma organização possa aprender. 0 desempenho do conhecimento pode ser explicado como a capacidade do indivíduo, grupo e organização para compreender o que aprenderam (KASSIM; SHOID 2013). O objetivo central dos estudos foi investigar determinantes significativos que correlacionem a CAO e Desempenho do conhecimento. A primeira pesquisa foi realizada com bibliotecários de uma universidade da Malásia tendo como universo - 36 respondentes. Os principais resultados apresentados nos três artigos indicam que:

a) alta correlação entre cultura organizacional e cooperação em trabalho de equipe (SHOID; KASSIM, 2012);

b) correlação moderada a forte existente entre as 4 dimensões (cultura organizacional, cooperação em trabalho de equipe, missão e visão compartilhada e pensamento sistêmico) e o desempenho do conhecimento. (KASSIM; SHOID, 2013);

c) relação moderada a positiva entre transferência de conhecimentos e liderança (SHOID; KASSIM, 2013);

d) relação moderada a positiva entre transferência de conhecimento e desempenho do conhecimento (SHOID; KASSIM, 2013);

e) fraca correlação entre liderança e desempenho conhecimento. (SHOID; KASSIM, 2013).

A segunda pesquisa foi realizada junto a bibliotecários que atuam em bibliotecas de diferentes universidades da Malásia. Os principais resultados apresentados nos dois artigos foram:

a) relação moderada a positiva entre habilidades e competências dos funcionários, tecnologias de Informação e comunicação - TIC e o desempenho do conhecimento (SHOID; KASSIM, 2014a); 
b) relação positiva e significativa entre as dimensões: missão e visão compartilhada, transferência de conhecimentos e o desempenho do conhecimento. (SHOID; KASSIM, 2014b).

Analisar a relação entre cultura da $A O$ e $O$ desempenho organizacional foi o objetivo de Yu e Chen (2012). Os resultados são apresentados em três artigos:

a) cultura de $\mathrm{AO}$ e desempenho organizacional - Principais resultados: as três dimensões da cultura organizacional "aprendizagem contínua", "incorporação", e "liderança" têm um impacto significativamente positivo sobre o desempenho do conhecimento organizacional. Além disso, tanto o "aprendizado contínuo" e "liderança" têm um impacto significativamente positivo sobre o desempenho organizacional extrínseco (YU; CHEN, 2012);

b) cultura da aprendizagem, métodos de aprendizagem e desempenho organizacional. As principais conclusões deste estudo foram: - o método de aprendizagem individual de "autoaprendizagem, utilizando recursos da Internet" é adotado frequentemente por funcionários da biblioteca em universidades e faculdades de Taiwan; - As duas dimensões mais fortes para a cultura de aprendizagem organizacional foram "promover instrução e diálogo" e "prover liderança estratégica para a aprendizagem." - as duas dimensões "criando oportunidades de aprendizagem contínua" e "criação de sistemas para capturar e compartilhar o aprendizado" podem influenciar os métodos de aprendizagem formal e informal, podendo contribuir positivamente para melhorar o desempenho do conhecimento das bibliotecas. Além disso, métodos de aprendizagem informal são susceptíveis para melhorar o desempenho do conhecimento (YU; CHEN, 2015);

c) cultura da aprendizagem individual, grupal e organizacional e AO. Principais resultados: - a percepção do valor da cultura de aprendizagem nos níveis individual, de equipe e organizacional não são fortes entre os funcionários da biblioteca em universidades e faculdades de Taiwan; - O estabelecimento de uma cultura de aprendizagem no nível organizacional é a chave para o desempenho eficaz de bibliotecas universitárias taiwanesas; - cultura de aprendizagem entre 0 indivíduo, a equipe e os níveis organizacionais têm uma relação positiva nas bibliotecas taiwanesas (YU, 2013). 
Os autores destacam que os líderes desempenham um papel-chave na organização formal de atividades de aprendizagem no trabalho para os funcionários da biblioteca e na criação de uma cultura de aprendizagem positiva para melhorar o desempenho de suas bibliotecas.

Estudos sobre práticas de aprendizagem em bibliotecas foram objetos de estudo de 3 pesquisas: Kassim e Nor (2007), Duarte et al. (2008) e Abdullah e Kassim, 2008.

Análise focada na $\mathrm{AO}$ em equipes foi o estudo desenvolvido por Kassim e Nor (2007) que examinou a percepção de bibliotecários acadêmicos que atuam em bibliotecas universitárias públicas e privadas sobre este tema. Os resultados demonstram que os bibliotecários acadêmicos percebem que aprendizagem no nível da equipe existe, mas eles não estão plenamente convencidos em que medida elas ocorrem. A percepção de práticas de aprendizagem no nível de equipe entre os respondentes que ocupam cargos de direção é mais positiva em relação aos que ocupam funções de gestão intermediária.

Duarte et al. (2008) objetivaram mapear práticas de AO em bibliotecas Universitárias. Trata-se de um estudo qualitativo, realizado com grupo focal como técnica. O artigo apresenta algumas práticas de aprendizagem individual e grupal e reforça a importância do uso do Grupo Focal como fonte geradora de novas ideias, como a criação de Comunidades de Prática entre as unidades de informação, assim como entre as bibliotecas universitárias do seu estado. Destacam, também, a relevância da criação de projetos integrativos organizacionais (ilhas de aprendizagem, qualificação e melhoria contínua, treinamentos especializados e integração entre grupos) no processo de desenvolvimento de $A O$.

A pesquisa de Abdullah e Kassim (2008) explora a percepção de bibliotecários sobre práticas de aprendizagem no nível organizacional com representantes de todas as universidades públicas e privadas do Yemen. Os resultados do estudo mostram que essas bibliotecas não podem ser consideradas como organizações de aprendizagem. Sugerem ampliação dos estudos sobre $\mathrm{AO}$ para entender as barreiras existentes para as práticas de $\mathrm{AO}$ e concluem que para sua efetivação a institucionalização da aprendizagem individual e de equipes são pré-requisitos

A análise do estilo de liderança transformacional e a sua relação com AO foi o objetivo de Dewi (2011), em sua pesquisa quantitativa com bibliotecários de instituições de ensino da Indonésia. Seu estudo concluiu que existe relação positiva entre $A O$ e $O$ estilo de Liderança Transformacional.

\subsection{Síntese dos resultados}

As pesquisas sobre $\mathrm{AO}$ em bibliotecas abrangeram temas diversos $\mathrm{e}$ os principais resultados apontam: 
a) que a cultura organizacional ocupa um papel preponderante nos processos de $\mathrm{AO}$, tanto nos níveis individual, como grupal e organizacional. Impacta positivamente no desempenho organizacional e é um elemento facilitador para aprendizagem a partir da absorção de estímulos externos;

b) que a liderança tem uma fraca correlação com desempenho do conhecimento, porém tem um impacto positivo significativo sobre o desempenho organizacional. 0 estilo de liderança transformacional tem uma relação positiva com AO;

c) que a aprendizagem individual é pré-requisito para $\mathrm{AO}$ e que o desenvolvimento de uma cultura de aprendizagem é essencial. A auto-aprendizagem com a utilização de recursos da internet é adotada frequentemente por bibliotecários de Universidades taiwanesas;

d) que a aprendizagem em equipe é pré-requisito para a $\mathrm{AO}$ e é influenciada pela cultura da organização. Essa cultura está altamente correlacionada com a cooperação em trabalho de equipe. Estudos demonstram que ela ocorre em bibliotecas, mas carece de mais estudos para saber em que medida isto acontece. Comunidade de aprendizagem (em redes intra e interdepartamentais) e comunidades de prática são fundamentais para gerar e transferir conhecimentos adquiridos;

e) AO e cultura de aprendizagem possuem uma relação positiva nas bibliotecas. Gestão da informação, do conhecimento e da aprendizagem organizacional estão altamente relacionados. A absorção de conhecimentos a partir da AI é baixa. As dimensões da cultura organizacional, aprendizagem e melhoria contínua, treinamentos e liderança têm um impacto significativamente positivo sobre 0 desempenho do conhecimento organizacional. Para efetivação da $\mathrm{AO}$, aprendizagem individual e de equipes são prérequisitos.

\section{Considerações finais}

Este artigo se propôs a realizar uma revisão sistemática em bases de dados nacionais e internacionais com o objetivo de identificar e analisar como o tema de $\mathrm{AO}$, relacionado ao contexto das bibliotecas acadêmicas, vem sendo abordado em estudos empíricos no período de 2005 a 2015. As análises bibliométricas constataram que a produção bibliográfica cresceu nos últimos cinco anos, sendo que a maior concentração de estudos ocorre em países do continente asiático. No Brasil, apenas um artigo foi localizado. O quadro das referências utilizadas indica uma 
tendência de estudos sobre bibliotecas como organizações de aprendizagem. As áreas temáticas abordadas nos estudos se concentram em: AO enquanto processo (aprendizagem individual, grupal e organizacional); relacionamentos da $A O$ com a GC, GI e Avaliação Institucional; impactos da Cultura organizacional sobre $A O$ e capacidade de aprendizagem organizacional. Quanto à abordagem metodológica utilizada prevalecem os estudos quantitativos com grandes populações e uso de questionários para coleta de dados. Nos estudos qualitativos a técnica mais utilizada foi a entrevista. Um estudo se diferenciou pela aplicação de Grupo Focal.

Os principais resultados apresentados nos artigos apontam sobre:

a) a relevância da cultura da aprendizagem, cultura organizacional e do estilo da liderança para a $\mathrm{AO}$ e o desempenho organizacional;

b) a importância da aprendizagem individual e grupal para se alcançar a $\mathrm{AO}$;

c) comunidade de aprendizagem (em redes intra e interdepartamental) e comunidades de prática são fundamentais para gerar e transferir conhecimentos adquiridos;

d) a absorção de conhecimentos a partir da AI é baixa, provavelmente causada por barreiras internas e fatores estruturais da AI.

Foram detectadas lacunas de estudos em bibliotecas acadêmicas relacionados aos seguintes temas: mudanças cognitivas e comportamentais; $A O$ e inovação, AO e memória organizacional e AO e desaprendizagem. Considera-se relevante que se amplie o quantitativo de estudos empíricos relacionados à $\mathrm{AO}$ em bibliotecas acadêmicas para a construção de novos conhecimentos. No tocante a realidade dentro das instituições brasileiras o campo de estudo encontra-se aberto, posto que somente um artigo foi encontrado nesta revisão. Visando dar continuidade a revisões sistemáticas sobre a temática, sugere-se ampliação de estudos para outras bases de dados, com tipologias de bibliotecas diversas, assim como envolvendo análises teórico-conceituais.

\section{Referências}

ABDULLAH, K. A. S.; KASSIM, N. A. Perceptions of organizational learning practices among Yemeni university librarians. Malaysian Journal of Library and Information Science, v. 13, n. 1, p. 77-90, 2008.

ANGELONI, M. T.; STEIL, A. V. Alinhamento de estratégias, aprendizagem e conhecimento organizacional. In: TARAPANOFF, K. Aprendizado 
organizacional: fundamentos e abordagens multidisciplinares. Curitiba: IBPEX, 2011. v. 1. p. 115-148.

ANTAL, A. B. et al. Organizational learning and knowledge: reflections on the dynamics of the field and challenges for the future. In: DIERKES, M. et al. (Org.). Handbook of organizational learning \& knowledge. Oxford: Oxford University Press, 2001. p. 921-939.

ANTONELLO, C. S. A metamorfose da aprendizagem organizacional: uma revisão crítica. In: RUAS, R.; ANTONELLO, C. S.; BOFF, L. H. (Orgs.). Aprendizagem organizacional e competências. Porto Alegre: Artmed, 2005. p. 12-33.

ANTONELLO, C. S.; GODOY, A. S. Aprendizagem organizacional no Brasil. Porto Alegre: Bookman, 2011.

ARGYRIS, C.; SCHÖN, D. Organizational learning: a theory of action perspective. Reading: Addison-Wesley, 1978.

BEM, R. M. de; COELHO, C. C. de S. R. Aplicações da gestão do conhecimento na área de biblioteconomia e ciência da informação: uma revisão sistemática. Brazilian Journal of Information Science, v. 7, n. 1, p. 69-97.

BONTIS, N.; CROSSAN, M.; HULLAND, J. Managing an organizational learning system by aligning stocks and flows. Journal of Management Studies, v. 39, n. 4, p. 438-469, 2002.

BOTELHO, L. L. R.; CUNHA, C. C. A.; MACEDO, M. O Método da revisão integrativa nos estudos organizacionais. Gestão e Sociedade, Belo Horizonte, v. 5, n. 11, p. 121-136, maio-ago. 2011.

CASEY, A.; OLIVERA, F. Organizational memory and forgetting. Journal of Management Inquiry, v. 20, n. 3, p. 305-310, 2011.

CHEN, K. Library evaluation and organizational learning: a questionnaire study. Journal of Librarianship end Information Science, v. 38, n. 2, p. 93$104,2006$.

CHEN, K. Institutional evaluation and its influence on organizational learning. Aslib Proceedings, v. 59, n.1, p. 5-25, 2007.

CHEN, K.; LIN, P. Organizational learning in the evaluation procedures: a qualitative study. College \& Research Libraries, v. 69, n. 1, p. 36-49, 2008.

CHIVA, R.; ALEGRE, J.; LAPIEDRA, R. Measuring organizational learning capability among the workforce. International Journal of Manpower, v. 28, n. 3, p. 224-242, 2007.

COSTA, M. D.; CASTRO, G. de. Gestão do conhecimento em bibliotecas no Brasil: um mapeamento temático com base na literatura técnico-científica. Seminário Nacional de Bibliotecas Universitárias, 17., 2014. Anais... Belo Horizonte: Universidade Federal de Minas Gerais, 2014. p. 1-14. 
CROSSAN, M. et al. Organizational learning: dimensions for a theory. The International Journal of Organizational Analysis, v. 3, n. 4, p. 337-360, 1995.

CROSSAN, M.; LANE, H.; WHITE, R. An organizational learning framework: from intuition to institution. Academy of Management Review, v. 24, n. 3, p. 522-537, 1999.

DEWI, I. C. Organizational learning and transformational leadership in higher education. In: RIBIERE; WORASINCHAI (Ed.). INTERNATIONAL CONFERENCE ON INTELLECTUAL CAPITAL, KNOWLEDGE MANAGEMENT AND ORGANISATIONAL LEARNING, 8., 2011. Proceedings... Bangkok, Thailand: IKI-SEA, 2011. v. 1. p. 149-156.

DUARTE, E. N. et al. Aprendizagem organizacional em unidades de informação: do grupo focal à comunidade de prática. Perspectivas em Ciência da Informação, v. 13, n. 3, p, 78-95, 2008.

EASTERBY-SMITH, M. Disciplines of organizational learning: contributions and critiques. Human Relations, v. 50, n. 9, p. 1085-113, 1997.

EASTERBY-SMITH, M.; LYLES, M. (Eds.). Handbook of organizational learning and knowledge management. Malden: Blackwell, 2005. p. 1-15.

EVALD, P. Organizational development in Danish public libraries: Computer mediated work and strategic vision. Journal of Librarianship and Information Science, v. 24, n. 3, p. 159-168, 1992.

FIGUEROA, L. A; GONZALEZ, A. B. Management of knowledge, information and organizational learning in university libraries. LIBRI, $\mathrm{V}$. 56, n. 3, p. 180-190, 2006.

FOWLER, R. K. The university library as learning organization for innovation: an exploratory study. College and Research Libraries, v.59, n. 3, p. 220-231, 1998.

GARVIN, A. Building a learning organisation. Harvard Business Review, v. 71, n. 4, p. 78-84, 1993.

GOH, S. C.; RICHARDS, G. Benchmarking the learning capability of organisations. European Management Journal, v. 15, n. 5, 575-583, 1997.

INKPEN, A.; CROSSAN, M. Believing is seeing: joint ventures and organizational learning. Journal of Management Studies, v. 32, n. 5, p. 595-618, 1995.

JANTZ, R. Knowledge management in academic libraries: special tools and processes to support information professionals. Reference Services Review, v. 29, n. 1, p. 33-39, Feb. 2001.

KASSIM, N. A.; NOR, A. M. Team learning in a learning organization: the practices of team learning among university librarians in Malaysia. Malaysian Journal of Library and Information Science, v. 12, n. 1, p. 5564, 2007. 
KASSIM, N. A.; SHOID, M. S. M. Organizational learning capabilities and knowledge performance in universiti teknologi Mara (UiTM) Library, Malaysia. World Applied Sciences Journal, v. 21, n. 1, p. 93-97, 2013.

KNIGHT, L. Network learning: exploring learning by interorganizational networks. Human Relations, v. 55, n. 4, p. 427-454, 2002.

LAVE, J.; WENGER, E. Situated learning: legitimate peripheral participation. New York: Cambridge University Press, 1991.

LEVINSTON, N.; ASAHI, M. Cross-national alliances and interorganizational learning. Organizational Dynamics, v. 24, n. 2, p. 5064, 1995.

LUBISCO, N. M. L. Bibliotecas universitárias, seus serviços e produtos: transposição de um modelo teórico de avaliação para um instrumento operacional - relatório de pesquisa I. PontodeAcesso Revista do Instituto de Ciência da Informação da UFBA, v. 8, p. 80-141, 2014.

PERISSÉ, A. R. S.; GOMES, M. da M.; NOGUEIRA, S. A. Revisões sistemáticas (inclusive metanálises) e diretrizes clínicas. In: GOMES, M. da M. (Org.). Medicina baseada em evidências: princípios e práticas. Rio de Janeiro (RJ): Reichmann \& Affonso, 2001. p. 131-48.

POLANYI, M. The tacit dimension. Gloucester: Peter Smith, c1966.

RIBEIRO JR., D. I. ; COSTA, M. P. P. Gestão do Conhecimento em unidades de informação: um modelo de retenção e compartilhamento de conhecimento apoiado por tecnologia de Wikis Semânticas. In: CONGRESSO BRASILEIRO DE BIBLIOTECONOMIA DOCUMENTAÇÃO E CIENCIA DA INFORMAÇÃO, 25., 2013. Anais... Florianópolis: FEBAB, 2013. p. 1406-1419.

ROWLEY, J. The library as a learning organization. Library Management, v. 18 , n. 2, p. 88-91, 1997.

SANTOS, J. L. S.; STEIL, A. V. Organizational learning and power dynamics: a study in a Brazilian University. Learning Organization, v. 22, p. 115-130, 2015.

SHANHONG, T. Knowledge management in libraries in the 21st century. In: IFLA COUNCIL AND GENERAL CONFERENCE, 66., 2000, Jerusalem, Israel. Anais... Jerusalém, Israel: IFLA, 2000. Disponível em: <http://www.ifla.org/IV/ifla66/papers/057-110e.htm>. Acesso em: 7 mar. 2004.

SHOID, M. S. M.; KASSIM, N. A. Organizational culture and teamwork cooperation as determinants of organizational learning capabilities (OLC) in academic library. In: ISBEIA 2012 - IEEE Symposium on Business, Engineering and Industrial Applications. 2012. [Anais...] Bandung, Indonesia, RMI, 2012. p. 274-278.

SHOID, M. S. M.; KASSIM, N. A. Ascertaining Dimensions of Organizational Learning Capabilities (OLC) in academic library. 
International Journal of Academic Research in Business and Social Sciences, v. 3, n. 7, p. 546-554, 2013.

SHOID, M. S. M.; KASSIM, N. A. Exploring the effect of organizational learning capabilities (OLC) on knowledge performance. World Applied Sciences Journal, v. 29, n. 12, p. 1544-1549, $2014 a$.

SHOID, M. S. M.; KASSIM, N. A. Using regression analysis on measuring organizational learning capabilities (OLC) dimensions. Middle - East Journal of Scientific Research, v. 19, n. 5, p. 615-619, 2014b.

SNYDER, W; CUMMINGS, T. Organizational learning disorders: conceptual model and intervention hypotheses. Human Relations, v. 51, n. 7, p. 873895, 1998.

SOUZA, I. M. DE Gestão das Universidades Federais Brasileiras: uma abordagem fundamentada na gestão do conhecimento. 399f. 2009. Tese (Doutorado em Engenharia e Gestão do Conhecimento) - Universidade Federal de Santa Catarina, Centro Tecnológico, Programa de PósGraduação em Engenharia e Gestão do Conhecimento, Florianópolis, 2009.

STEIL, A. V.; SANTOS, J. L. S. Building conceptual relations between organizational learning, knowledge, and memory. International Journal of Business \& Management Tomorrow, v. 2, p. 1-9, 2012.

STEIL, A V. Um modelo de aprendizagem organizacional baseado na ampliação de competências desenvolvidas em programas de capacitação. 218f. 2002. Tese (Doutorado em Engenharia) - Programa de Pósgraduação em Engenharia de Produção, Universidade Federal de Santa Catarina, Florianópolis, 2002.

TAKAHASHI, A. R. W. Descortinando os processos da aprendizagem organizacional no desenvolvimento de competências em instituições de ensino. 467f. 2007. Tese (Doutorado em Administração) - Universidade de São Paulo, São Paulo, 2007.

TSANG, E. W. K. Organizational learning and the learning organization: a dichotomy between descriptive and prescriptive research. Human Relations, v. 50, n. 1, p. 73-89, 1997.

TSANG, E. W. K.; ZAHRA, S. Organizational unlearning. Human Relations, v. 6, n. 10, p. 1435-1462, 2008.

YU, T. The relationships among individual, team and organizational learning in Taiwan's university and college libraries. In: IFLA WLIC Future Libraries: infinite possibilities - in Session 100 - Continuing Professional Development and Workplace Learning with Education and Training. 2013. Proceedings... Singapura: IFLA, 2013. p. 1-15.

YU, T.; CHEN, C. Study on the relationship between organizational learning culture and organizational performance in Taiwan's university and 
college libraries. Journal of Educational Media and Library Science, v. 50, n. 2, p. 173-200, 2012.

YU, T.; CHEN, C. The relationship of learning culture, learning method, and organizational performance in the university and college libraries in Taiwan. LIBRI, v. 65, n. 1, p. 1-14, 2015.

ZAHRA, S. A.; GEORGE, G. Absorptive capacity: a review, reconceptualization, and extension. Academy of Management Review, $\mathrm{v}$. 27, n. 2, p. 185-203, Apr. 2002.

marouva.faqueti@gmail.com

joao.bosco.mota.alves@ufsc.br

andreasteil@egc.ufsc.br 